\title{
A NONCOMMUTATIVE ALGORITHM FOR MULTIPLYING $3 \times 3$ MATRICES USING 23 MULTIPLICATIONS
}

\author{
BY JULIAN D. LADERMAN
}

Communicated by Jacob Schwartz, October 9, 1975

A noncommutative algorithm, as opposed to a commutative algorithm, performs matrix multiplication without requiring the matrix elements to be commutative under the operation of multiplication. Such an algorithm is most desirable since it can be employed to multiply two matrices whose elements are themselves matrices.

The standard definition for the multiplication of two $n \times n$ matrices yields a noncommutative algorithm using $n^{3}$ multiplications. Strassen [7] produced a noncommutative algorithm which multiplies two $2 \times 2$ matrices using seven multiplications. In that paper Strassen proved that two $n \times n$ matrices could be multiplied asymptotically in $O\left(n^{\log _{2} 7}\right) \sim O\left(n^{2.81}\right)$ multiplications and likewise $O\left(n^{\log _{2} 7}\right)$ total arithmetic operations. He accomplished this by embedding $n \times n$ matrices into larger matrices $\left(m 2^{k} \times m 2^{k}\right)$ and then employing recursively his $2 \times 2$ algorithm. In that procedure the noncommutativity of the algorithm was essential.

Since $O\left(n^{2}\right)$ arithmetic operations is the best asymptotic lower bound for multiplying two $n \times n$ matrices and many problems are related to matrix multiplication (see Strassen [7] and Munro [6]) much interest has developed in finding better algorithms. Winograd [9] proved that seven multiplications are required by any algorithm for the product of two $2 \times 2$ matrices.

In multiplying $3 \times 3$ matrices, since $\log _{2} 7 \sim \log _{3} 21.8$, a noncommutative algorithm requiring 21 or fewer multiplications is needed to improve on Strassen's asymptotic result. In [3] Gastinel produced a noncommutative algorithm using 25 multiplications. Hopcroft and Kerr [4] described the construction of a noncommutative algorithm with 24 multiplications and Hopcroft and Musinski [5] explicitly state several such algorithms. Following is a noncommutative algorithm using 23 multiplications for obtaining $C=A B$ where $A$ and $B$ are $3 \times 3$ matrices. Obviously the number of additions in this algorithm could be greatly reduced, but it is being given in its more basic form.

Let

$A M S(M O S)$ subject classifications (1970). Primary 68A10, 65F30; Secondary 68A20, $65 \mathrm{H} 10$. 


$$
\begin{aligned}
& m_{1}=\left(a_{11}+a_{12}+a_{13}-a_{21}-a_{22}-a_{32}-a_{33}\right) b_{22}, \\
& m_{2}=\left(a_{11}-a_{21}\right)\left(-b_{12}+b_{22}\right) \text {, } \\
& m_{3}=a_{22}\left(-b_{11}+b_{12}+b_{21}-b_{22}-b_{23}-b_{31}+b_{33}\right) \text {, } \\
& m_{4}=\left(-a_{11}+a_{21}+a_{22}\right)\left(b_{11}-b_{12}+b_{22}\right) \text {, } \\
& m_{5}=\left(a_{21}+a_{22}\right)\left(-b_{11}+b_{12}\right) \text {, } \\
& m_{6}=a_{11} b_{11} \text {, } \\
& m_{7}=\left(-a_{11}+a_{31}+a_{32}\right)\left(b_{11}-b_{13}+b_{23}\right) \text {, } \\
& m_{8}=\left(-a_{11}+a_{31}\right)\left(b_{13}-b_{23}\right) \text {, } \\
& m_{9}=\left(a_{31}+a_{32}\right)\left(-b_{11}+b_{13}\right) \text {, } \\
& m_{10}=\left(a_{11}+a_{12}+a_{13}-a_{22}-a_{23}-a_{31}-a_{32}\right) b_{23} \text {, } \\
& m_{11}=a_{32}\left(-b_{11}+b_{13}+b_{21}-b_{22}-b_{23}-b_{31}+b_{32}\right) \text {, } \\
& m_{12}=\left(-a_{13}+a_{32}+a_{33}\right)\left(b_{22}+b_{31}-b_{32}\right) \text {, } \\
& m_{13}=\left(a_{13}-a_{33}\right)\left(b_{22}-b_{32}\right) \text {, } \\
& m_{14}=a_{13} b_{31} \text {, } \\
& m_{15}=\left(a_{32}+a_{33}\right)\left(-b_{31}+b_{32}\right) \text {, } \\
& m_{16}=\left(-a_{13}+a_{22}+a_{23}\right)\left(b_{23}+b_{31}-b_{33}\right) \text {, } \\
& m_{17}=\left(a_{13}-a_{23}\right)\left(b_{23}-b_{33}\right) \text {, } \\
& m_{18}=\left(a_{22}+a_{23}\right)\left(-b_{31}+b_{33}\right) \text {, } \\
& m_{19}=a_{12} b_{21} \text {, } \\
& m_{20}=a_{23} b_{32} \text {, } \\
& m_{21}=a_{21} b_{13} \text {, } \\
& m_{22}=a_{31} b_{12} \text {, } \\
& m_{23}=a_{33} b_{33} \text {. }
\end{aligned}
$$

Then

$$
\begin{aligned}
& C_{11}=m_{6}+m_{14}+m_{19}, \\
& C_{12}=m_{1}+m_{4}+m_{5}+m_{6}+m_{12}+m_{14}+m_{15}, \\
& C_{13}=m_{6}+m_{7}+m_{9}+m_{10}+m_{14}+m_{16}+m_{18}, \\
& C_{21}=m_{2}+m_{3}+m_{4}+m_{6}+m_{14}+m_{16}+m_{17}, \\
& C_{22}=m_{2}+m_{4}+m_{5}+m_{6}+m_{20},
\end{aligned}
$$




$$
\begin{aligned}
& C_{23}=m_{14}+m_{16}+m_{17}+m_{18}+m_{21}, \\
& C_{31}=m_{6}+m_{7}+m_{8}+m_{11}+m_{12}+m_{13}+m_{14}, \\
& C_{32}=m_{12}+m_{13}+m_{14}+m_{15}+m_{22}, \\
& C_{33}=m_{6}+m_{7}+m_{8}+m_{9}+m_{23} .
\end{aligned}
$$

The well-known commutative algorithms of Winograd [8] involve 24 multiplications in the $3 \times 3$ case. Brockett and Dobkin [2] produced a commutative algorithm using 23 multiplications without counting multiplications and divisions by 2 .

The algorithm in this paper was produced by finding an integer solution to the following system ${ }^{1}$ of 729 nonlinear algebraic equations involving 621 unknowns:

$$
\sum_{t=1}^{23} z_{i k t} x_{g h t} y_{r s t}=\delta_{i g} \delta_{h r} \delta_{s k} \quad \text { where } i, k, g, h, r, s=1,2,3 .
$$

No use of computers was made in solving this system of equations. It was obtained by merging the solutions of four smaller systems of equations with similar but fewer terms. A detailed explanation of the merging procedure will appear elsewhere. Similar systems of equations and merging procedures may produce algorithms which improve on Strassen's asymptotic result.

\section{REFERENCES}

1. R. Brent, Algorithms for matrix multiplication, Technical Report STAN-CS-70-157, Dept. of Computer Science, Stanford Univ., 1970.

2. R. Brockett and D. Dobkin, On the number of multiplications required for matrix multiplication, SIAM J. Comput. (to appear).

3. N. Gastinel, Sur le calcul des produits de matrices, Numer. Math. 17 (1971), 222229. MR $45 \# 4616$.

4. J. Hopcroft and L. Kerr, On minimizing the number of multiplications necessary for matrix multiplication, SIAM J. Appl. Math. 20 (1971), 30-36. MR 43 \#58.

5. J. Hopcroft and J. Musinski, Duality applied to the complexity of matrix multiplication and other bilinear forms, SIAM J. Comput. 2 (1973), 159-173.

6. I. Munro, Problems related to matrix multiplication, Proc. Courant Inst. Sympos. Computational Complexity, 1971, pp. 137-151.

7. V. Strassen, Gaussian elimination is not optimal, Numer. Math. 13 (1969), 354356. MR 40 \#2223.

8. S. Winograd, $A$ new algorithm for inner product, IEEE Trans. Computers 17 (1968), $693-694$. $381-388$.

9. - On multiplication of $2 \times 2$ matrices, Linear Algebra and Appl. 4 (1971), 10468

DEPARTMENT OF MATHEMATICS, LEHMAN COLLEGE, BRONX, NEW YORK

COURANT INSTITUTE OF MATHEMATICAL SCIENCES, NEW YORK UNIVERSITY, NEW YORK, NEW YORK 10012

1 These equations were brought to my attention by my dissertation advisor, Professor Peter Ungar. At that time we felt these equations were original but later it was found that Brent [1] had studied this system. 